\title{
Propagation des incertitudes en modélisation hydraulique des rivières
}

\author{
Adil Fahsi, Azzeddine Soulaïmani ${ }^{1 *}$ Georges W. Tchamen ${ }^{2}$ \\ ${ }^{1}$ École de technologie supérieure (ÉTS), Montréal,H3C1K3 Canada, e-mail : adil.fahsi.1@ens.etsmtl.ca,e-mail : azzeddine.soulaimani@etsmtl.ca \\ ${ }^{2}$ Hydro-Québec, Unité Barrages et Hydraulique, Montréal, H2Z 1A4 Canada, e-mail :Tchamen.Georges_Williams@hydro.qc.ca
}

\begin{abstract}
RÉSUMÉ. - Dans le domaine de l'ingénierie hydraulique, la prise en compte de la variabilité des paramètres de l'écoulement est devenue incontournable dans une démarche de conception robuste. La simulation de Monte Carlo reste l'outil le plus fiable pour la détermination de la probabilité de défaillance. Elle reste cependant très coûteuse surtout pour les systèmes complexes comportant des modèles éléments finis ou volumes finis de grandes tailles et ayant de nombreux paramètres de conception incertains. D'autre part, l'utilisation des méthodes économiques basées sur l'indice de fiabilité nécessite le calcul du vecteur gradient et de la matrice Hessienne de la fonction d'état limite (Rackwitz-Fiessler, Newton). Ce calcul est très délicat lorsque la fonction d'état limite est définie à partir d'un modèle numérique ne fournissant que des approximations de la solution exacte du problème, ce qui peut entraîner des erreurs ou des non convergences dans certains cas. Ce travail se situe dans le cadre d'un couplage entre les simulations numériques en hydrodynamique des rivières et les méthodes fiabilistes basées sur l'indice de fiabilité. Nous proposons une méthode dite " quasi-Newton, BFGS » en introduisant dans l'algorithme de Newton une procédure d'évaluation de la matrice Hessienne en tenant compte de la précision numérique du résultat sur la fonction d'état limite. La méthode proposée a été mise en œuvre et appliquée à un écoulement en rivière, l'Aisne en France. Les résultats obtenus, en particulier l'intervalle de confiance à $95 \%$ de la ligne d'eau, ont été comparés aux résultats de référence utilisant la simulation Monte Carlo. Ils ont permis de mettre en évidence la robustesse et l'efficacité de la méthodologie proposée et ont contribué à la compréhension de la défaillance en déterminant l'influence des paramètres incertains introduits à l'exercice de modélisation.
\end{abstract}

Mots clés : écoulements en rivière; incertitudes ; méthode de Monté Carlo ; FORM ; tirage d'importance.

\section{Propagation of uncertainties in rivers hydraulic modelling}

\begin{abstract}
In hydraulic engineering, the physical properties governing the flow are known up to some degrees of uncertainties. It is thus important for a safe and optimal design of hydraulic structures to evaluate the uncertainties propagation trough the system from the input data to the output results. The Monte Carlo simulation is the simplest and direct method to evaluate the probability of safety or of failure. However, for very small probabilities such evaluation can prove very prohibitive especially when using large-size finite elements of finite volume models with a large number of uncertain parameters. On the other hand, approximate reliability methods based on the reliability index such as FORM rely on the solution of an optimization problem. For fast and robust convergence, gradient-based iterative methods are the methods of choice. In this paper, we present a coupling procedure of a quasi-Newton-BFGS algorithm with FORM and Importance sampling reliability methods to evaluate small probabilities of failure. These techniques are applied to the case of a flow in the Aisne River (France) where Stickler's coefficient, the discharge and the bathymetry parameters are considered as the uncertain variables. The $95 \%$ confidence interval is then obtained for the water elevation. The results are compared with those obtained with the Monte Carlo simulations. These tests show the robustness and efficiency of the proposed methodology and highlight the most significant uncertain parameters in the modelling process.
\end{abstract}

Key words : fluvial flows ; uncertainties ; Monte Carlo method ; FORM ; importance sampling.

\section{INTRODUCTION}

Les méthodes numériques de discrétisation (par exemple les méthodes des éléments finis et des volumes finis) constituent un outil puissant pour modéliser les écoulements et concevoir les ouvrages hydrauliques. Elles permettent de discrétiser judicieusement les équations de conservation qui gouvernent ces écoulements. Les paramètres d'entrée à un modèle numérique, tels que le débit $[16,27]$ et les niveaux d'eau aux frontières, la bathymétrie et la topographie sous forme de modèle de terrain [14], ainsi que les coefficients

*. Corresponding author de frottement $[15,23,12,29]$, présentent naturellement des incertitudes. Celles-ci proviennent de la variabilité intrinsèque de ces paramètres (comme par exemple le coefficient de Strickler qui varie au sein d'un même bief du fait de l'hétérogénéité du fond), de l'erreur de mesure liée au dispositif utilisé ou à un manque de connaissance sur ces paramètres. De même, les actions climatiques appliquées aux ouvrages hydrauliques ne sont pas toujours parfaitement connues [7, 20]. Par conséquent, le couplage entre les méthodes fiabilistes et les calculs hydrauliques en utilisant les modèles aux éléments finis ou volumes finis devient une nécessité $[9,19,21]$. Durant les vingt dernières années, de nombreux travaux ont été consacrés à ce couplage [17]. Sur le plan 
théorique, les apports mathématiques ont permis de trouver des solutions. En pratique cependant, l'analyse probabiliste se heurte toujours à la question du temps de calcul, en effet, la prise en compte des incertitudes des paramètres physiques s'accompagne d'une inflation des temps de calculs lors des simulations [17,9]. Cet obstacle limite souvent l'application des méthodes fiabilistes. La question du développement d'outils permettant de minimiser le temps de calcul en assurant la précision des résultats reste donc d'actualité.

Un certain nombre de méthodes fiabilistes existent pour analyser l'impact des incertitudes des données d'entrée sur les variables d'intérêt. On peut citer la méthode de simulation de type Monte-Carlo [24], les méthodes basées sur la technique des surfaces de réponse [8], les méthodes variationnelles [3], les méthodes stochastiques basées sur le développement en polynômes du chaos [25], ainsi que les méthodes fiabilistes basées sur l'indice de fiabilité (de type FORM, SORM, tirage d'importance) [17]. Ces dernières méthodes permettent d'estimer la probabilité de défaillance à un coût numérique réduit. L'indice de fiabilité, noté $\beta$, est par ailleurs la mesure de fiabilité la plus fréquemment référencée dans les codes de conception et de dimensionnement. Cet indice est défini dans l'espace des variables aléatoires normales centrées réduites et indépendantes, appelé espace standardisé. L'indice $\beta$ est défini comme la distance minimale entre l'origine de l'espace gaussien et la surface d'état limite. Afin de déterminer l'indice de fiabilité, un problème d'optimisation non linéaire est ainsi posé. L'algorithme de Newton est un algorithme itératif robuste pour déterminer la solution de ce problème. Cet algorithme, ainsi que plusieurs de ses variantes, nécessitent le calcul du vecteur gradient et de la matrice Hessienne qui sont composés des dérivées partielles premières et secondes de la fonction d'état limite par rapport aux variables normées. Nous proposons, dans cette étude, une procédure simple et efficace pour évaluer la matrice Hessienne de la fonction d'état limite. Cette procédure permet de réduire le temps de calculs numériques et offre une convergence rapide.

Cet article propose une méthodologie générale pour estimer la propagation des incertitudes des paramètres d'entrée d'un modèle numérique en hydraulique. Cette méthodologie est appliquée au cas de la rivière l'Aisne en France. Les résultats obtenus, en particulier l'intervalle de confiance à $95 \%$ de la ligne d'eau, sont comparés avec ceux obtenus avec les simulations directes de Monté Carlo.

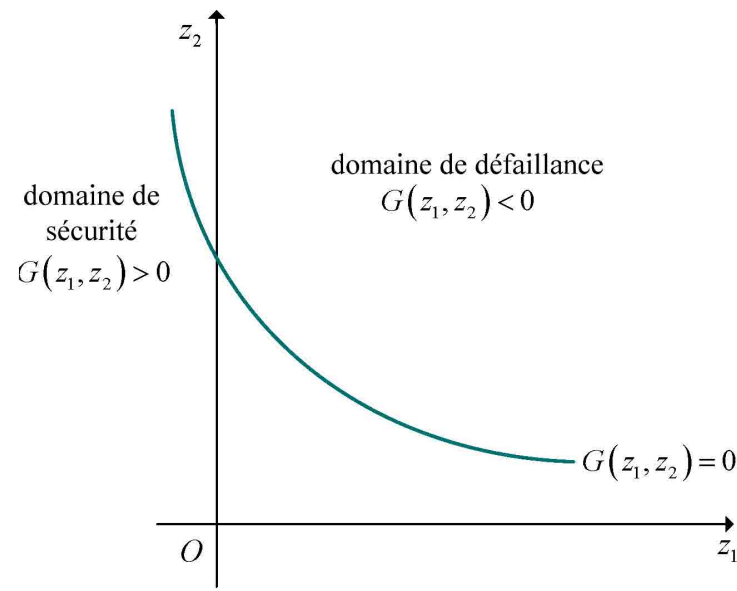

Espace phvsiaue

\section{MÉTHODES FIABILISTES BASÉES SUR L'INDICE DE FIABILITÉ}

Ces méthodes s'attachent à déterminer la probabilité de défaillance d'un système $\mathrm{G}(\mathrm{z})$ dans des cas où une simulation directe de Monte Carlo serait très difficile à mettre en œuvre. Pour ce faire, quelle que soit la densité de probabilité des variables physiques aléatoires $\mathrm{z}$, on se ramène toujours dans un espace où les variables (notées u) sont Gaussiennes, normées et centrées, c.-à-d. de moyenne nulle et d'écart type unitaire :

$$
T: z_{i}=F_{i}^{-1}\left(\Phi\left(u_{i}\right)\right)
$$

où $\mathrm{T}$ est appelée transformation isoprobabiliste, $F_{i}^{-1}$ est l'inverse de la fonction de répartition de la variable aléatoire $z_{i}$ et $\Phi$ est la fonction de répartition de la loi normale centrée réduite. La fonction d'état limite $\mathrm{G}(\mathrm{z})$, qui décrit le critère de défaillance, est transformée en la fonction $\mathrm{H}(\mathrm{u})$ dans l'espace normé (figure 1).

Dans ce nouvel espace, ces méthodes sont appliquées pour se rapprocher de l'état limite. Classiquement la probabilité de défaillance est estimée par l'intermédiaire du calcul de l'indice de fiabilité $\beta$ de Hasofer-Lind [13]. En effet on cherche le point $\mathrm{u}^{*}$, appelé point de conception. Géométriquement l'indice de fiabilité est défini comme étant le minimum de la distance de l'origine de l'espace des variables normées construit à la fonction d'état limite $\mathrm{H}(\mathrm{u})$, ce qui donne :

$$
\beta=-{ }^{T} u^{*} \frac{\nabla H\left(u^{*}\right)}{\left\|\nabla H\left(u^{*}\right)\right\|}
$$

Connaissant alors le point de conception, on peut évaluer la probabilité de défaillance :

- par une approximation FORM du premier ordre [13] : en remplaçant la fonction d'état limite par un hyperplan tangent au point $\mathrm{u}^{*}$ (figure 1 ).

$$
P_{f}=P(G(z) \leq 0) \approx \Phi(-\beta)
$$

L'égalité précédente est exacte pour le cas où les variables aléatoires de base sont gaussiennes avec un état limite linéaire.

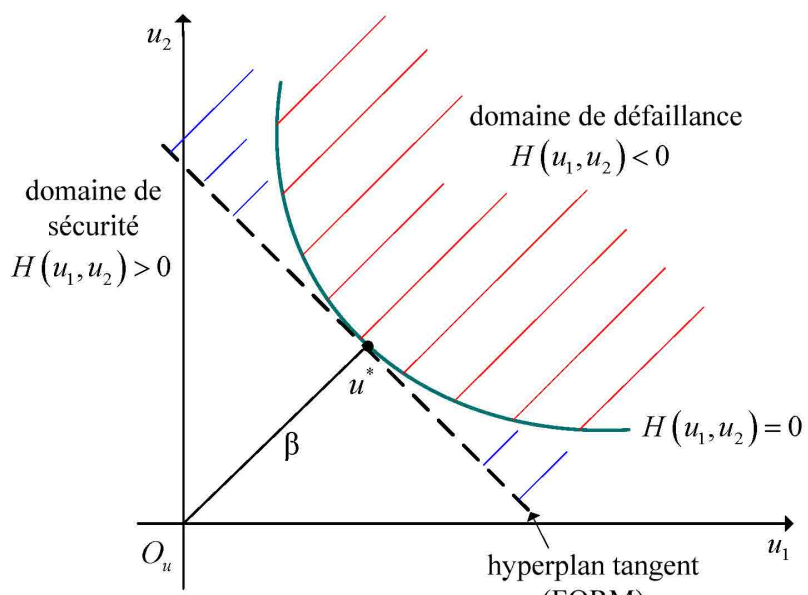

(FORM)

Esbace gaussien centré et réduit

Figure 1 : Définition de l'indice de fiabilité et de l'approximation FORM. 
- par la méthode de tirage d'importance (IS) [18] : en réalisant des tirages au voisinage du point de conception $\mathrm{u}^{*}$ pour améliorer l'évaluation de la probabilité de défaillance obtenue par la méthode FORM. À partir de N tirages indépendants suivant une densité de tirage gaussienne centrée sur $\mathrm{u}^{*}$ et réduite, la probabilité de défaillance est estimée par [17]:

$$
P_{f} \approx \frac{1}{N} \sum_{i=1}^{N}\left(I_{(G(z) \leq 0)}^{(i)} \exp \left(-{ }^{T} u^{*} u^{(i)}-\frac{\beta^{2}}{2}\right)\right)
$$

Pour l'évaluation de la probabilité de défaillance à l'aide des ces méthodes, on doit auparavant évaluer l'indice de fiabilité $\beta$. Ce qui se traduit par un problème d'optimisation sous la contrainte suivante : trouver le point de conception $\mathrm{u}^{*}$ associé aux paramètres de la fonction d'état limite dans l'espace normé telle que $\mathrm{H}(\mathrm{u})=0$, qui minimise la distance $\mathrm{Ou}^{*}$.

Dans ce contexte, Plusieurs algorithmes ont été développés pour trouver efficacement $\mathrm{u}^{*}$. Citons celui de RackwitzFiessler (HLRF) qui est basé sur le calcul des gradients de chaque variable [22]. Le point de défaillance le plus probable est obtenu par itérations successives lorsque les précisions souhaitées sur la fonction d'état limite et sur deux points consécutifs de l'algorithme sont obtenues, soit pour deux itérations successives k et $\mathrm{k}+1:\left|H\left(u^{(k)}\right)\right| \leq \varepsilon_{H}$ et $\left\|u^{(k+1)}-u^{(k)}\right\| \leq \varepsilon_{u}$.

L'indice $\beta^{(k+l)}$ est calculé par (2) à partir du vecteur $u^{(k+1)}:$

$$
\begin{gathered}
u^{(k+1)}=\left(u^{(k)} \alpha^{(k)}+\frac{H\left(u^{(k)}\right)}{\left\|\nabla H\left(u^{(k)}\right)\right\|}\right) \alpha^{(k)} \\
\alpha^{(k)}=-\frac{\nabla H\left(u^{(k)}\right)}{\left\|\nabla H\left(u^{(k)}\right)\right\|}
\end{gathered}
$$

Un des intérêts de FORM réside dans la possibilité d'accéder aux cosinus directeurs $\alpha_{i}$ qui nous renseignent sur la sensibilité, au point de conception, de l'indice de fiabilité $\beta$ par rapport à chaque variable aléatoire normée $u_{i}$.

\section{ALGORITHME QUASI-NEWTON (BFGS)}

L'algorithme Rackwitz-Fiessler est l'algorithme de Newton le plus simple, on émet l'hypothèse que la dérivée seconde de la fonction d'état limite est négligeable. La convergence globale de cet algorithme n'est pas assurée, et il faut souvent essayer plusieurs points de départ pour obtenir la convergence vers un point optimal. C'est un algorithme très utilisé dans les codes commerciaux.

Avec les méthodes quasi-Newton, on généralise la formule itérative de Newton. Le principe de ces méthodes de résolution est de générer une séquence de matrices symétriques qui soient des approximations, toujours améliorées, de la matrice dérivée seconde (Hessienne) de la fonction d'état limite.

$$
\begin{gathered}
u^{(k+1)}=u^{(k)}-\mathrm{B}^{(k)}\left(2 u^{(k)}+\lambda^{(k+1)} \nabla H\left(u^{(k)}\right)\right) \\
\mathrm{B}^{(k)}=\left[2 \mathrm{I}+\lambda^{(k)} \nabla^{2} H\left(u^{(k)}\right)\right]^{-1}
\end{gathered}
$$

$$
\lambda^{(k+1)}=\lambda^{(k)}+\frac{H\left(u^{(k)}\right)-\nabla H\left(u^{(k)}\right)^{T} \mathrm{~B}^{(k)}\left(2 u^{(k)}+\lambda^{(k)} \nabla H\left(u^{(k)}\right)\right)}{\nabla H\left(u^{(k)}\right)^{T} \mathrm{~B}^{(k)} \nabla H\left(u^{(k)}\right)}
$$

où $\lambda^{(k)}$ est le multiplicateur de Lagrange à l'itération $\mathrm{k}$, et $\mathrm{I}$ est la matrice identité.

Entre la multitude des algorithmes quasi-Newton, la différence principale est donc le choix d'approximation de la matrice Hessienne et sa mise à jour. La formule de correction qui permet d'obtenir $\tilde{\nabla}^{2} H\left(u^{(k+1)}\right)$ à partir de $\tilde{\nabla}^{2} H\left(u^{(k)}\right)$ utilise des informations nouvelles obtenues lors de l'itération $\mathrm{k}+1$ :

$$
\tilde{\nabla}^{2} H\left(u^{(k+1)}\right)=\tilde{\nabla}^{2} H\left(u^{(k)}\right)+W^{(k)}
$$

Actuellement, l'unanimité est faite autour d'une formule trouvée indépendamment par Broyden, Fletcher, Goldfard et Shanno (BFGS) [2] :

$$
\begin{gathered}
W^{(k)}=\frac{y^{(k)} y^{(k) T}}{y^{(k) T} d^{(k)}}-\frac{\tilde{\nabla}^{2} H\left(u^{(k)}\right) d^{(k)} d^{(k) T} \tilde{\nabla}^{2} H\left(u^{(k)}\right)}{d^{(k) T} \tilde{\nabla}^{2} H\left(u^{(k)}\right) d^{(k)}} \\
d^{(k)}=u^{(k+1)}-u^{(k)} \quad \text { et: } \quad y^{(k)}=\nabla H\left(u^{(k+1)}\right)-\nabla H\left(u^{(k)}\right)
\end{gathered}
$$

La direction $d^{(k)}$ (12) n'est pas forcément une direction de descente, elle l'est si la matrice $\mathrm{B}^{(k)}(8)$ est définie positive $\left(v_{i}^{(k)} \geq 0\right.$ pour $i=1, \ldots, n$ avec $v_{i}^{(k)}=i^{\text {eme }}$ valeur propre de $\mathrm{B}^{(k)}$ ), pour contourner ce problème deux solutions ont été proposées :

- Prendre $\tilde{\nabla}^{2} H\left(u^{(k)}\right)=\tilde{\nabla}^{2} H\left(u^{(k-1)}\right)$;

- Prendre $\tilde{\nabla}^{2} H\left(u^{(k)}\right)=0$ et appliquer l'algorithme RackwitzFiessler à l'itération suivante.

Dans un premier temps, nous avons opté pour la première solution.

\section{APPLICATION}

Les algorithmes détaillés précédemment ont été programmés et couplés avec le logiciel de modélisation hydraulique monodimensionnelle Mascaret [11].

L'exemple de l'étude se situe sur la rivière de l'Aisne (France) entre la commune de Soissons (02) et la commune de Vic-sur-Aisne (02) sur une distance d'environ $13 \mathrm{~km} \mathrm{[6].}$ L'Aisne est un affluent de l'Oise qu'elle rejoint à la commune de Compiègne, c'est une rivière de plaine aux crues lentes. Le tronçon étudié est homogène, il peut donc être assimilé à un bief, il est décrit par 20 profils. Les profils sont repérés par rapport à l'axe d'écoulement au moyen d'abscisses curvilignes qui suivent les courbes de la rivière. Ces abscisses sont mesurées selon des points kilométriques ou pK. Le tronçon ainsi décrit va du pK 0 au pK 13038,97m. Le lit mineur est caractérisé par une largeur au miroir de 50 à $80 \mathrm{~m}$ tandis que le lit majeur a une largeur variable comprise entre 800 et $1200 \mathrm{~m}$. La figure 3 montre le profil en long très irrégulier du fond du tronçon étudié.

Le logiciel Mascaret est un code de volumes finis de modélisation hydraulique monodimensionnelle à surface libre basé sur les équations de Saint-Venant. La modélisation hydraulique $1 \mathrm{D}$ est très pertinente pour des écoulements débordant peu du lit mineur [28]. Les résultats restent satisfaisants lorsque le débordement est important, à condition que la plaine d'inondation ne présente pas de trop fortes 
singularités, comme des obstacles transversaux par exemple, et que la topologie de la rivière n'évolue pas avec le débit.

L'enjeu est alors d'assurer un dialogue entre les deux outils numériques :

- code probabiliste : définition des réalisations du vecteur des variables aléatoires $\mathrm{z}$, transformation isoprobabiliste, validation des pas de calcul et de la convergence pour l'obtention du point de conception $\mathrm{u}^{*}$, calcul des indicateurs de fiabilité ;

- code volumes finis : évaluations de $\mathrm{G}$ et de ses gradients.

Pour réaliser ce couplage, on choisi un couplage direct [17] dans lequel le code fiabiliste fait appel au code volumes finis pour chaque évaluation de la fonction d'état limite, définie par:

$$
G(z)=h_{0}-g(z), \text { avec: } z=\left(z_{1}, z_{2}, \ldots, z_{13}\right)
$$

où $h_{0}$ indique le seuil fixé pour la ligne d'eau et $\mathrm{g}(\mathrm{z})$ le niveau de la ligne d'eau définie de façons discrète en tout point du maillage par le code Mascaret. La défaillance se produit quand : $h_{0} \leq g(z)$.

Nous avons utilisé le régime permanent et nous imposons un débit à l'entrée et un niveau à la sortie à chaque itération (courbe de tarage programmée sur le logiciel Matlab).

Le pas du maillage est de $30 \mathrm{~m}$ et le pas de planimétrie est de $0.33 \mathrm{~m}$ (découpage en tranche horizontale des profils en travers).

\section{IV.1. Données hydrologiques : débit}

Entre sa source et la fin de son parcours, l'Aisne a traversé $353 \mathrm{~km}$ et reçu une série d'affluents. La saison des hautes eaux s'étend désormais de décembre à début mai pour des débits mensuels moyens allant de 85 à $120 \mathrm{~m}^{3} / \mathrm{s}$, avec un maximum en février. La saison des basses eaux se produit de juillet à octobre avec un minimum de $24 \mathrm{~m}^{3} / \mathrm{s}$ au mois d'août. Le débit moyen annuel est de $65.40 \mathrm{~m}^{3} / \mathrm{s}$. À la station Vic-sur-Aisne (aval de la zone étudiée), les QJ ou débits calculés pour une crue journalière sont: QJ5 $=302 \mathrm{~m}^{3} / \mathrm{s}$ et $\mathrm{QJ} 50=500 \mathrm{~m}^{3} / \mathrm{s}$ [26]. Ces niveaux sont bien suffisants pour occasionner de sérieux débordements.

Nous effectuons une analyse basée sur le débit QJ50, la référence [5] suggère que le coefficient de variation (ou l'incertitude autour de ce débit moyen) est de l'ordre de $15 \%$ $\left(c_{v}=\sigma / \mu\right)$. En l'absence d'information suffisante pour ajuster finement les lois de probabilités des variables incertaines d'entrée, on en est réduit à utiliser la loi normale. Les caractéristiques (moyenne $\mu$ et écart-type $\sigma$ ) sont rassemblées dans le tableau 1. Ces valeurs sont déduites des références trouvées sur la rivière de l'application, l'Aisne en France $[26,6,5]$. La méthodologie suivie peut-être utilisée pour d'autres lois de probabilité.

\section{IV.2. Paramètres de calage : coefficients de Strickler}

La rugosité, représentée le plus souvent par le coefficient de Strickler, est un paramètre à la fois difficile à évaluer et influent sur les résultats de calculs. La pratique montre que pour les modèles $1 \mathrm{D}$, les variations relatives du coefficient de Strickler, en fonction de l'observateur sur le terrain, et des calages des simulations sont d'environ $15 \%$ à $30 \%$ [5]. Cet écart pouvant notamment varier en fonction de la taille du site et de la résolution spatiale adoptée (nombre de biefs).
Le code Mascaret permet de considérer des coefficients de Strickler différents pour le lit mineur et le lit majeur. Il existe un certain nombre d'approches qui fournissent des ordres de grandeur du coefficient de Strickler en fonction du type de cours d'eau étudié, la plus utilisée étant celle de M. Pardé [4], cette référence suggère que : $k s_{\min }=35 \mathrm{~m}^{1 / 3} / \mathrm{s}$ et $k s_{m a j}=12 \mathrm{~m}^{1 / 3} / \mathrm{s}$. Nous supposons une loi normale tronquée pour représenter la distribution du coefficient de Strickler. Les bornes de variation ont été fixées à dire d'expert $[10,15]$.

\section{IV.3. Données bathymétriques}

Les données bathymétriques ne sont accessibles que par levés terrestres avec ou sans contact. Ces levés sont incontournables lorsque des simulations numériques, 1D ou 2D, sont à effectuer. Des profils en long et en travers sont souvent levés en zone inondable pour servir de contrôle. La précision des levés des données bathymétriques est souvent donnée pour environ $20 \mathrm{~cm}$ pour les parties immergées [5].

Le traitement des données de la bathymétrie peut être facilité si nous ne retenons que des entiers, les bornes des intervalles de $1 \mathrm{~m}\left[b_{i-1}, b_{i}\left[\right.\right.$, avec : $b_{i}=31,32, \ldots, 40 \mathrm{~m}$. Plus précisément, chaque section en travers est représentée par l'ensemble des segments passant par les niveaux $b_{i}$. Un exemple d'une section en travers modifiée par l'interpolation linéaire est décrit à la figure 2 . Les sections modifiées sont utilisées dans les calculs de l'écoulement. L'interpolation linéaire est utilisée afin de faciliter la manipulation de la variable aléatoire de la bathymétrie. Dix niveaux (de 31 à $40 \mathrm{~m}$ ) sont enfin retenus dans la représentation aléatoire de la bathymétrie de tout le domaine. Nous supposons que ces variables suivent une distribution normale.

Ainsi, outre le débit et les coefficients de Strickler du lit majeur et mineur, on a 10 variables aléatoires représentant les incertitudes sur la bathymétrie, soit au total 13 variables aléatoires. En l'absence d'information suffisante nous faisons l'hypothèse que ces variables sont indépendantes.

\section{RÉSULTATS}

L'analyse probabiliste est effectuée à l'aide des méthodes FORM et tirage d'importance, les algorithmes BFGS et HLRF sont utilisés pour la résolution du problème d'optimisation.

Les tolérances de convergence sont $\varepsilon_{H}=0.001$ et $\varepsilon_{u}=0.01$ pour les algorithmes BFGS et HLRF et $c_{v} \leq 0.1$ pour la méthode de tirage d'importance (IS). Il convient de vérifier

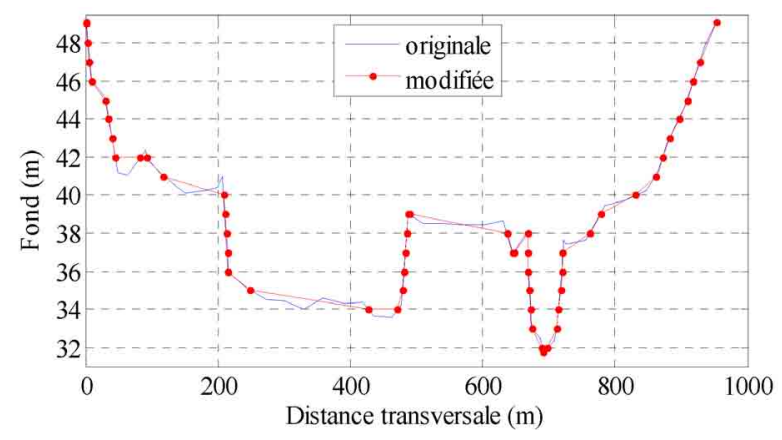

Figure 2 : Exemple d'une section originale du bassin et son interpolation linéaire. 
Tableau 1 : Description des variables aléatoires d'entrée.

\begin{tabular}{|c|c|c|c|c|c|c|c|}
\hline \multicolumn{2}{|c|}{ Variable } & Loi de distribution & $\mu$ & $\boldsymbol{\sigma}$ & $c_{v}$ & $\operatorname{Max}$ & Max \\
\hline \multicolumn{2}{|c|}{$\mathrm{z}_{1}:$ débit QJ50 $\left(\mathrm{m}^{3} / \mathrm{s}\right)$} & normale & 500 & 75 & $15 \%$ & 300 & 700 \\
\hline \multicolumn{2}{|c|}{$\mathrm{z}_{2}:$ Strickler lit mineur $\left(\mathrm{m}^{1 / 3} / \mathrm{s}\right)$} & normale & 35 & 7 & $20 \%$ & 22 & 48 \\
\hline \multicolumn{2}{|c|}{$\mathrm{z}_{3}:$ Strickler lit majeur $\left(\mathrm{m}^{1 / 3} / \mathrm{s}\right)$} & normale & 12 & 4 & $33 \%$ & 5 & 19 \\
\hline \multirow{4}{*}{ 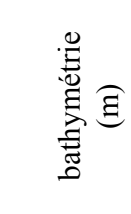 } & $\mathrm{z}_{4}:$ ligne 31 & normale & 31 & 0.31 & $1 \%$ & 30.50 & 31.50 \\
\hline & $\mathrm{z}_{5}$ : ligne 32 & normale & 32 & 0.32 & $1 \%$ & 31.50 & 32.50 \\
\hline & $\vdots$ & $\vdots$ & & & $\vdots$ & & \\
\hline & $\mathrm{z}_{13}:$ ligne 40 & normale & 40 & 0.40 & $1 \%$ & 39.50 & 40.50 \\
\hline
\end{tabular}

les résultats issus de ces méthodes par des calculs de type Monte Carlo $\left(\mathrm{MC}, c_{v}=\sqrt{\left(1-P_{f}\right) / N P_{f}} \leq 0.1\right)$.

\section{V.1. Comparaison de l'algorithme proposée BFGS avec l'algorithme HLRF}

Les critères de comparaison entre les deux algorithmes sont la valeur de l'indice de fiabilité $\beta$, les coordonnées du point de conception $\mathrm{u}^{*}$, la valeur de la fonction d'état limite au point $\mathrm{u}^{*} \mathrm{G}\left(\mathrm{u}^{*}\right)$, qui vaut zéro si $\mathrm{u}^{*}$ se trouve exactement sur la surface de défaillance, et le nombre de calculs de la fonction d'état limite $\mathrm{N}$ (indicateur du temps de calcul associé à la méthode). Les résultats obtenus sont présentés dans le tableau 2. L'indice de fiabilité $\beta$ et les coordonnées $\mathrm{du}$ point $\mathrm{u}^{*}$ obtenus par les deux algorithmes sont similaires. La proximité du point $\mathrm{u}^{*}$ à la surface de défaillance est satisfaisante dans les deux algorithmes. BFGS est tou- tefois l'algorithme la plus efficace en termes de nombre d'appels au calcul de la fonction d'état limite. L'indice de fiabilité estimé par BFGS converge vers une valeur stable après 8 itérations.

\section{V.2. Intervalle de confiance à $95 \%$ de la ligne d'eau}

Nous avons considéré différents point de discrétisation $\mathrm{x}$ pour lesquels on a obtenu le quantile à $2.5 \%$, nous avons fixé la probabilité de défaillance à $P_{f}=0.025$ et nous avons cherché les seuils de la ligne d'eau $h_{0}$ correspondants (13). Les résultats obtenus sont présentés dans le tableau 3. La méthode d'approximation FORM et la méthode IS, même avec un nombre important de variables aléatoires (13 variables), fournissent de très bons résultats. Les résultats ont été comparés avec succès aux résultats de MC. Les imprécisions observées correspondent à des erreurs numériques lors

Tableau 2: Résultats obtenus $\left(h_{0}=41.6 \mathrm{~m}, x=5728.58 \mathrm{~m}\right)$.

\begin{tabular}{|l|c|c|c|c|c|c|c|c|}
\hline Algorithme & $\boldsymbol{\beta}$ & $u_{1}^{*}$ & $u_{2}^{*}$ & $u_{3}^{*}$ & $u_{4}^{*}$ & $u_{5}^{*}$ & $u_{6}^{*}$ & $u_{7}^{*}$ \\
\hline HLRF & 2.547 & 1.462 & -1.448 & -1.477 & 0.026 & 0.067 & 0.135 & 0.077 \\
\hline BFGS & 2.536 & 1.459 & -1.462 & -1.465 & 0.025 & 0.070 & 0.134 & 0.077 \\
\hline
\end{tabular}

\begin{tabular}{|l|c|c|c|c|c|c|c|c|}
\hline Algorithme & $u_{8}^{*}$ & $u_{9}^{*}$ & $u_{10}^{*}$ & $u_{11}^{*}$ & $u_{12}^{*}$ & $u_{13}^{*}$ & $\mathbf{G}\left(\mathbf{u}^{*}\right)$ & $\mathbf{N}$ \\
\hline HLRF & 0.048 & 0.042 & 0.071 & 0.106 & 0.132 & 0.067 & 0.0001 & $\mathbf{2 8 0}$ \\
\hline BFGS & 0.051 & 0.042 & 0.070 & 0.108 & 0.137 & 0.070 & 0.001 & $\mathbf{1 1 2}$ \\
\hline
\end{tabular}

Tableau 3 : Valeurs du quantile à $2.5 \%$ de la ligne d'eau calculées par différentes méthodes.

\begin{tabular}{|c|c|c|c|c|c|c|}
\hline Méthode & $P_{f}$ & $x=1999.95 \mathrm{~m}$ & $x=5728.58 \mathrm{~m}$ & $x=7924.72 \mathrm{~m}$ & $x=12194.60 \mathrm{~m}$ & Temps \\
\hline FORM & 0.025 & $\begin{array}{c}h_{0} \approx 41.88 \mathrm{~m} \\
196 \text { appels à } \mathrm{G}\end{array}$ & $\begin{array}{c}h_{0} \approx 41.16 \mathrm{~m} \\
182 \text { appels à } \mathrm{G}\end{array}$ & $\begin{array}{c}h_{0} \approx 40.72 \mathrm{~m} \\
112 \text { appels à } \mathrm{G}\end{array}$ & $\begin{array}{c}h_{0} \approx 39.92 \mathrm{~m} \\
196 \text { appels à } \mathrm{G}\end{array}$ & $0.66 \%$ \\
\hline IS & 0.025 & $\begin{array}{c}h_{0} \approx 41.89 \mathrm{~m} \\
1152 \text { appels à } \mathrm{G}\end{array}$ & $\begin{array}{c}h_{0} \approx 41.17 \mathrm{~m} \\
1724 \text { appels à } \mathrm{G}\end{array}$ & $\begin{array}{c}h_{0} \approx 40.75 \mathrm{~m} \\
1570 \text { appels à } \mathrm{G}\end{array}$ & $\begin{array}{c}h_{0} \approx 39.94 \mathrm{~m} \\
1192 \text { appels à } \mathrm{G}\end{array}$ & $4.70 \%$ \\
\hline $\mathrm{MC}$ & 0.025 & $\begin{array}{c}h_{0}=41.80 \mathrm{~m} \\
3 \times 10^{4} \text { appels à } \mathrm{G}\end{array}$ & $\begin{array}{c}h_{0}=41.10 \mathrm{~m} \\
3 \times 10^{4} \text { appels à } \mathrm{G}\end{array}$ & $\begin{array}{c}h_{0}=40.68 \mathrm{~m} \\
3 \times 10^{4} \text { appels à } \mathrm{G}\end{array}$ & $\begin{array}{c}h_{0}=39.89 \mathrm{~m} \\
3 \times 10^{4} \text { appels à } \mathrm{G}\end{array}$ & $100 \%$ \\
\hline
\end{tabular}




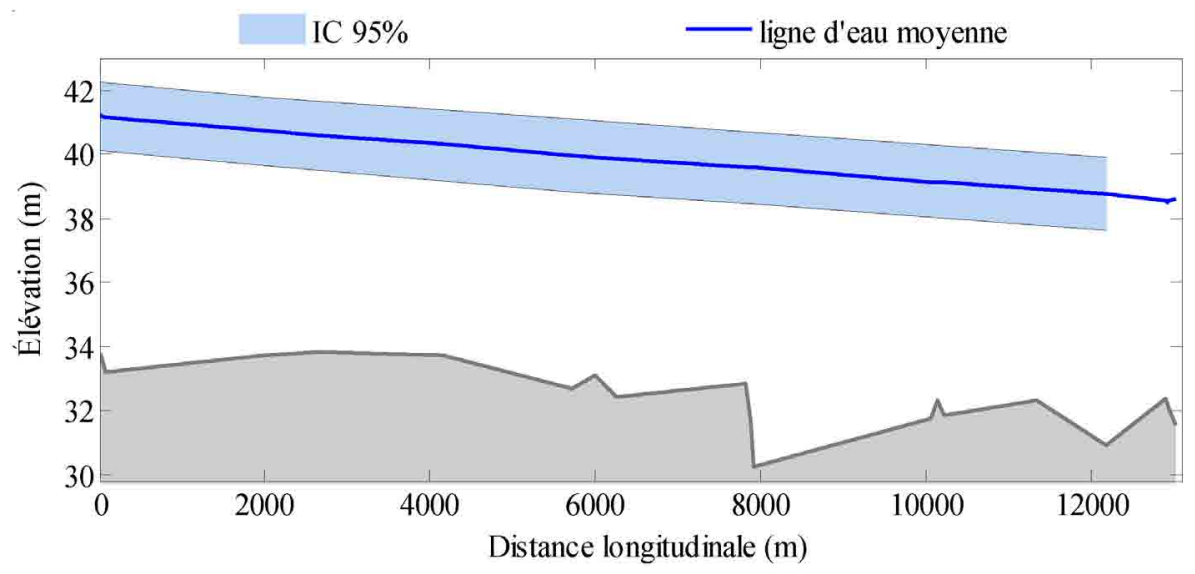

Figure 3 : Ligne d'eau moyenne basée sur le débit journalier cinquantenaire et l'intervalle de confiance à $95 \%$ (quantiles à $2.5 \%$ et à $97.5 \%$ calculés par la méthode $M C$ ).

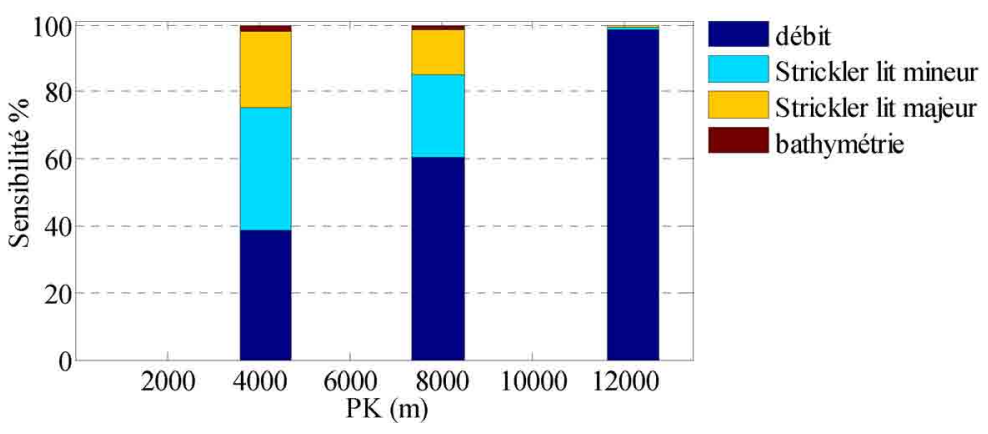

Figure 4 : Hiérarchisation des sources d'incertitudes (FORM, $\left.P_{f}=0.025\right)$.

de la recherche du point de conception et à la courbure de la fonction d'état limite en ce point.

Le calcul nécessite un nombre d'évaluations de la fonction d'état limite G. Le tableau 3 montre que la méthode MC est la plus coûteuse $\left(3 \times 10^{4}\right.$ simulations qui ont pris $\left.8 \mathrm{~h}\right)$, la méthode d'approximation FORM a un temps d'exécution raisonnable (quelques minutes).

$\mathrm{Au}$ regard des bons résultats fournis par la méthode FORM, nous avons choisi de retenir cette approche dans l'analyse de sensibilité. Cette démarche, propre aux études fiabilistes, permettra de calculer l'influence de chaque variable aléatoire sur la probabilité de défaillance.

La figure 4 présente les poids respectifs des variables considérées sur la probabilité de défaillance en trois points de discrétisation $\times(4148 \mathrm{~m}, 7925 \mathrm{~m}$ et $12195 \mathrm{~m})$. Le débit

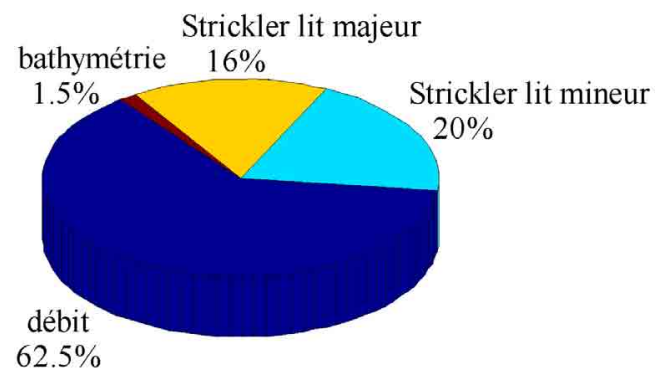

Figure 5 : Valeurs moyennes des sensibilités (FORM, $\left.P_{f}=0.025\right)$. et les coefficients de Strickler jouent un rôle important, suivis dans une moindre mesure par la bathymétrie. Mais cette importance relative varie avec la distance, les coefficients de Strickler du lit mineur et du lit majeur semblent avoir beaucoup d'influence au début de l'écoulement, notamment dans la première moitié du site. Les valeurs moyennes des sensibilités sur tout le tronçon étudié sont présentées sur la figure 5 .

\section{CONCLUSION}

L'objectif principal de cette recherche était d'apporter une contribution à la réalisation du couplage entre les méthodes fiabilistes et les simulations numériques en hydrodynamique des rivières. La méthode BFGS permet d'utiliser efficacement l'algorithme d'ordre deux de Newton, même si le modèle volumes finis associé à la fonction d'état limite ne permet qu'une certaine précision lors de l'estimation des dérivées partielles par différences finies. Les résultats de l'application ont bien montré l'intérêt de la méthode proposée : la convergence reste facile et rapide, le point de conception est situé à une très bonne proximité de la surface d'état limite et les résultats de la probabilité de défaillance ont été comparés avec succès aux résultats de Monte Carlo. L'analyse de sensibilité apporte également un éclairage sur l'importance de certaines variables aléatoires en modélisation hydrodynamique sur la fiabilité. Les résultats de ce couplage sont très encourageants et ouvrent la perspective de leurs applications pour établir les cartes d'inondation correspondantes à des seuils de probabilité de dépassement. 


\section{RÉFÉRENCES}

[1] Breitung K. (1984) - Asymptotic approximations for multinormal integrals. Journal of Engineering Mechanics Division ASCE. 110(3) 357-366

[2] Broyden C.G. (1970) — The Convergence of a Class of Double-rank Minimization Algorithms. Journal of the Institute of Mathematics and its Applications. 6 76-90

[3] Sudret B. \& Der Kiureghian A. (2000) - Stochastic Finite Element Methods and Reliability. A State-of-the-Art Report. Report No.UCB/SEMM-2000/08, Department of Civil \& Engineering University of California, Berkeley.

[4] CARLIER M. (1982) - Hydraulique générale et appliquée. Eyrolles (Paris)

[5] Cetmef. (2001) - Étude de sensibilité des modélisations 1D. Rapport technique du CETMEF/DVNE. Département Chaussées, Pont, Hydraulique, CETE Méditerranée (France). $67 \mathrm{p}$

[6] Chasse P., Descatoire I., \& Weil B. (2007) - Guide de prise en main Fudaa-Mascaret 2.0. Centre d'études techniques maritimes et fluviales (CETMEF). $145 \mathrm{p}$

[7] Darlymple T. (1960) — Flood Frequency Analysis, in Water Supply Paper. US Geological Survey.

[8] De Rocquigney E. (2005) - Couplage mécano-probabiliste pour la fiabilité des structures - un cas industriel où la robustesse d'une surface de réponse est démontrable. Actes du $17^{\mathrm{e}}$ Congrès français de mécanique, Troyes.

[9] De Rocquigny E., Devictor N., \& Tarantola S. (2008) Uncertainty in Industrial Practice - A guide to quantitative uncertainty management. Wiley Press.

[10] DuN R.W. (2006) - Reducing uncertainty in the hydraulic analysis of canals. Water Management. 159(4)

[11] Goutal N., \& Maurel F. (2002) - A finite volume solver for $1 \mathrm{D}$ shallow water equations applied to an actual river. International Journal for Numerical Methods in Fluids. 38 1-19

[12] Goutal N., Bernardara P., De Rocquigny E., \& Arnaud A (2008) - Uncertainties in 1D flood level modeling: stochastic analysis of upstream discharge and friction parameter influence. Floodsite final meeting. Oxford.

[13] Hasofer A. M., \& Lind N. C. (1974) - An exact and invariant first order reliability format. Journal of Engineering Mechanics. 100 111-121

[14] Horrit M.S. (2002) — Stochastic modelling of 1-D shallow water flows over uncertain topography. Journal of Computational Physics. 180 327-338
[15] JoHnSON P.A. (1996) - Uncertainty of Hydraulic Parameters. Journal of Hydraulic Engineering. 122(2) 112-114

[16] KLemes V. (2000) - Tall tales about tails of hydrological distributions. Journal of Hydrologic Engineering. 5(3) 227-239

[17] Lemaire M., Chateauneuf A. \& Mitteau J. C. (2005) Fiabilité des structures: couplage mécano-fiabiliste statique. Lavoisier - Hermès. $506 \mathrm{p}$

[18] Melchers R. E. (1989) — Importance sampling in structural systems. Structural Safety. 6 3-10

[19] Merwade, V., Olivera F., Arabi M., \& Edleman S. (2008) Uncertainty in Flood Inundation Mapping : Current Issues and Future Directions. Journal of Hydrologic Engineering. 13(7) 608-620

[20] Miquel J. (1984) - Guide pratique d'estimation des probabilités des crues. Collection de la Direction des études et Recherches d'EDF. 166 p.

[21] Pappenberger F., \& Beven K. (2006) - Ignorance is bliss. 7 reasons not to use uncertainty analysis. Water Resource Research. 42(W05302)

[22] Rackwitz R., \& Fiessler B. (1979) - Structural reliability under combined random load sequences. Computers and Structures. 9 489-494

[23] ROBERT A. (1990) - Boundary roughness in coarse grained channel. Progress in Physical Geography. 14 42-70

[24] Rubinstein R. Y. (1981) - Simulation and the Monte-Carlo Method. John Wiley and Sons.

[25] Solaiman B. (2006) — Processus stochastiques pour l'ingénieur. Presses Polytechniques et Universitaires Romandes.

[26] Tison C. (2007) - Marché d'assistance à maîtrise d'ouvrage pour la détermination des données hydrologiques au droit des barrages dans le cadre du projet de reconstruction des barrages des bassins de l'Aisne et de la Meuse et de leur équipement en microcentrales, Etude relative au bassin versant de l'Aisne.Rapport technique de Voies Navigables de France, BCEOM - HEN 60764Z. Béthune 62408. 105 p

[27] VAN Gelder P.H.A.J.M., \& MAI C.V. (2008) — Distribution functions of extreme sea waves and river discharges. Journal of Hydraulic Research. 46(2) 280-291

[28] Weber J., \& MenÉndez A. (2004) - Performance of lateral velocity distribution models for compound channel sections. Actes de colloque, River Flow 2004, Second International conference on Fluvial Hydraulics, Naples. $1449-457$

[29] Wohl E.E. (1998) - Uncertainty in Flood Estimates Associated with Roughness Coefficient. Journal of Hydraulic Engineering. 124(2) 219-223 